\title{
Seasonal variations in the reported incidence of sexually transmitted diseases in Scotland (1972-76)
}

\author{
C. B. S. SCHOFIELD \\ From Newcastle General Hospital, Newcastle upon Tyne
}

SUMMARY The seasonal variation in the quarterly incidence of some sexually transmitted diseases and other conditions in Scotland is compared with that of gonorrhoea and of conceptions leading to live births or abortions. The seasonal incidence for non-specific genital infections and for other conditions not requiring treatment in both men and women was similar to that of gonorrhoea, thus indicating an association with promiscuity, whereas the seasonal variation for candidosis in men and women-an infection not usually associated with promiscuity-was similar to that of conceptions. Differences between the sexes occurred, however, in the seasonal incidences of scabies, pubic lice, genital herpes, trichomoniasis, and other conditions requiring treatment; thus men with these conditions appeared to be more promiscuous than women. The seasonal variations in incidence of genital scabies and pubic lice indicate that these infestations are more easily transmitted by close bodily contact indoors during cold weather than in the open air.

\section{Introduction}

Most venereologists in Britain are well aware that the busiest month in their clinics is August. Cornelius (1971) reported that the quarterly incidence of gonorrhoea in the United States was 94, 97, 110, and $100 \%(100 \%=$ that expected if attendance was the same in each quarter). Similar seasonal variations have been noted in the reported incidence of gonorrhoea in Scotland but not in that of all the other sexual:y transmitted diseases nor in the quarterly incidence of abortions or live births.

About $65 \%$ of gonococcal infections in Scotland are acquired as a result of indiscriminate, promiscuous, sexual intercourse (Schofield, 1975). This finding has formed the basis for a hypothesis that the seasonal variations in the reported incidence of the venereal diseases (those mainly acquired as a result of indiscriminate promiscuity) would be similar to those of gonorrhoea-given a fairly constant incubation period-whereas the non-venereal diseases (those not usually associated with promiscuity) would have seasonal variations in incidence similar to those of conceptions leading to live births or abortions.

Address for reprints: Dr C. B. S. Schofield, Ward 34, Newcastle General Hospital, Westgate Road, Newcastle upon Tyne NE4 6BE

Received for publication 10 July 1978
Method

STATISTICS

Information on the number of patients attending special clinics was obtained from the quarterly statistical returns (ISD(D)5) submitted to the Scottish Home and Health Department for the years 1972-76 inclusive. Live birth statistics were obtained from the annual report of the Registrar General for Scotland (1976) and the abortion statistics from the Scottish Home and Health Department's (1973-76) published figures, in both cases for the years 1971-76 inclusive. To estimate the time of conceptions leading to abortions the published figures were adjusted back $2 \frac{1}{2}$ months, the average duration of pregnancies before therapeutic abortions being between 10 and 11 weeks, while those for live births were adjusted back nine months. The quarterly incidences are recorded as percentages of those expected, had an equal number of patients attended each quarter (Figure) and the annual pattern is repeated to show the continuity between the fourth and first quarters.

\section{Results}

\section{GONORRHOEA}

The seasonal incidence of genital gonorrhoea in men and women was similar, with the maximum attendance in the third quarter and the minimum in 

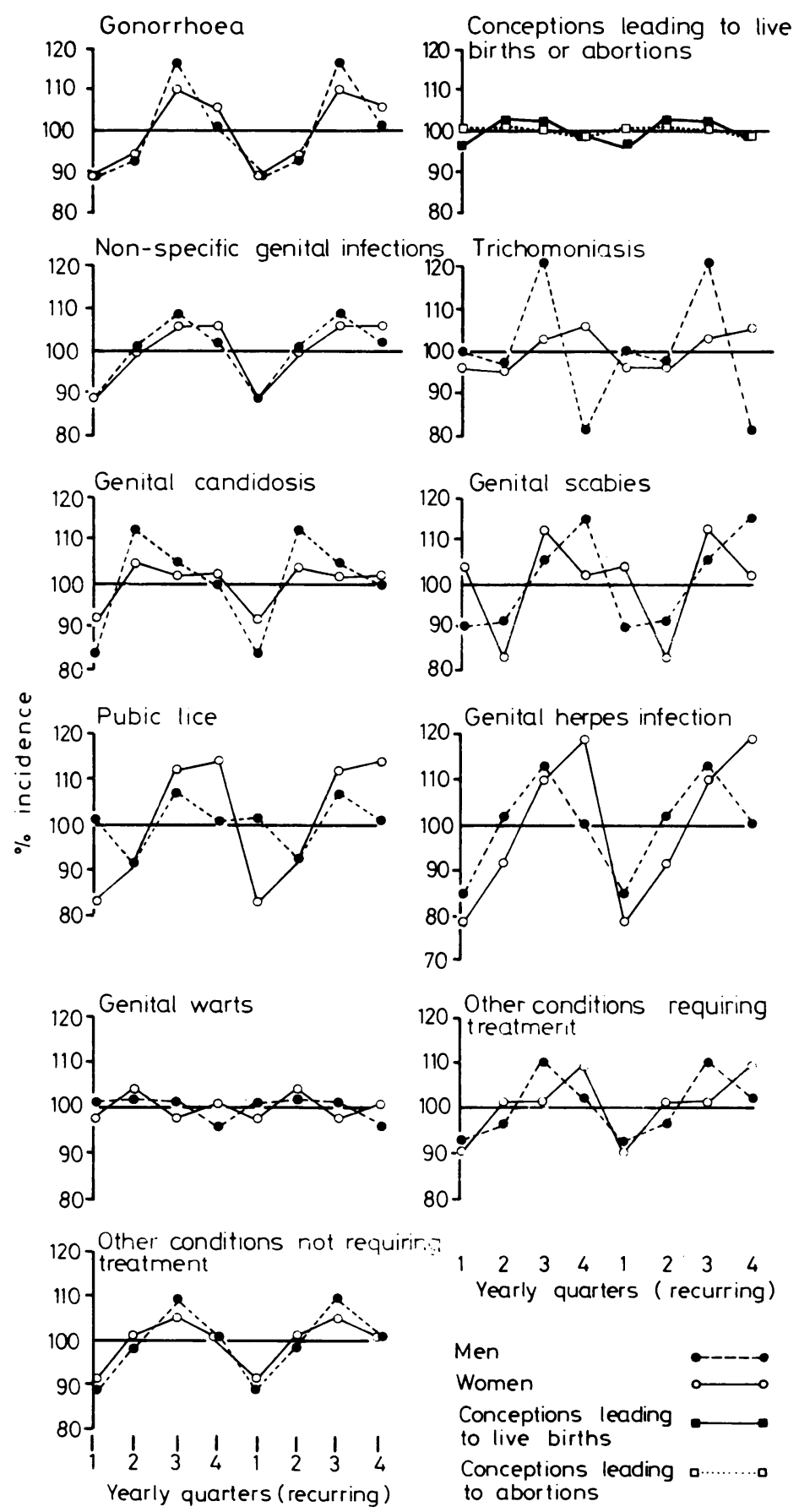

Figure Percentage quarterly incidence of gonorrhoea, conceptions leading to live births or abortions, non-specific genital infections, trichomoniasis, candidosis, scabies, pubic lice, herpes, warts, other conditions requiring treatment, and other conditions not requiring treatment. (The annual pattern is repeated to show the continuity between the fourth and first quarters.) 
the first, the ranges being $27 \cdot 8 \%$ and $20 \cdot 8 \%$ respectively (Figure and Tables 1 and 2).

\section{CONCEPTIONS}

The seasonal incidence of conceptions leading to live births was similar to that of conceptions leading to therapeutic abortions (Figure and Tables 1 and 2). The maximum incidence of both occurred in the second quarter decreasing to a minimum in the fourth, while the ranges were only $5 \cdot 5 \%$ and $2 \cdot 8 \%$ respectively, thus giving different patterns from those of gonorrhoea.

\section{NON-SPECIFIC GENITAL INFECTIONS}

The seasonal incidence of non-specific genital infections (Figure) was similar to that of gonorrhoea, with the maximum attendance in the third quarter and the minimum in the first, the ranges being $19 \cdot 7 \%$ and $16 \cdot 8 \%$ respectively (Table 1 ).

\section{TRICHOMONIASIS}

The number of men with trichomoniasis was very small (Table 1), but the seasonal incidence was an exaggeration of that of gonorrhoea, with a range of $39.9 \%$ (Figure). The seasonal incidence of trichomoniasis in women differed from that of gonorrhoea and of conceptions, with the maximum attendance in the fourth quarter and the minimum in the second, the range being $10 \cdot 3 \%$.

\section{CANDIDOSIS}

The seasonal incidence of genital candidosis in men and women was similar and quite unlike that of gonorrhoea (Figure). With maximum attendance in the second quarter and minimum in the first (ranges of $29 \cdot 6 \%$ and $13 \cdot 2 \%$ respectively) the patterns were exaggerations of those of conceptions (Table 1).

\section{SCABIES}

Only 169 cases of genital scabies in women were recorded over the five-year period (Figure and Table 1). Maximum attendance was in the third quarter but another peak occurred in the first, the range being $28 \cdot 5 \%$. The seasonal incidence in men-with minimum attendance in the first two quarters and maximum in the fourth-did not resemble that of gonorrhoea or of conceptions, the range being $24 \cdot 6 \%$ (Table 1 ).

PUBIC LICE

Few cases of pubic lice occurred in women (Figure and Table 1); the minimum attendance occurred during the first quarter and the maximum in the third and fourth, with a range of $30.9 \%$. The seasonal incidence of pubic lice in men was similar to that of gonorrhoea, with minimum attendance in the second quarter, the range being $14 \cdot 5 \%$.

Table 1 Number of attendances by men and women with sexually transmitted diseases

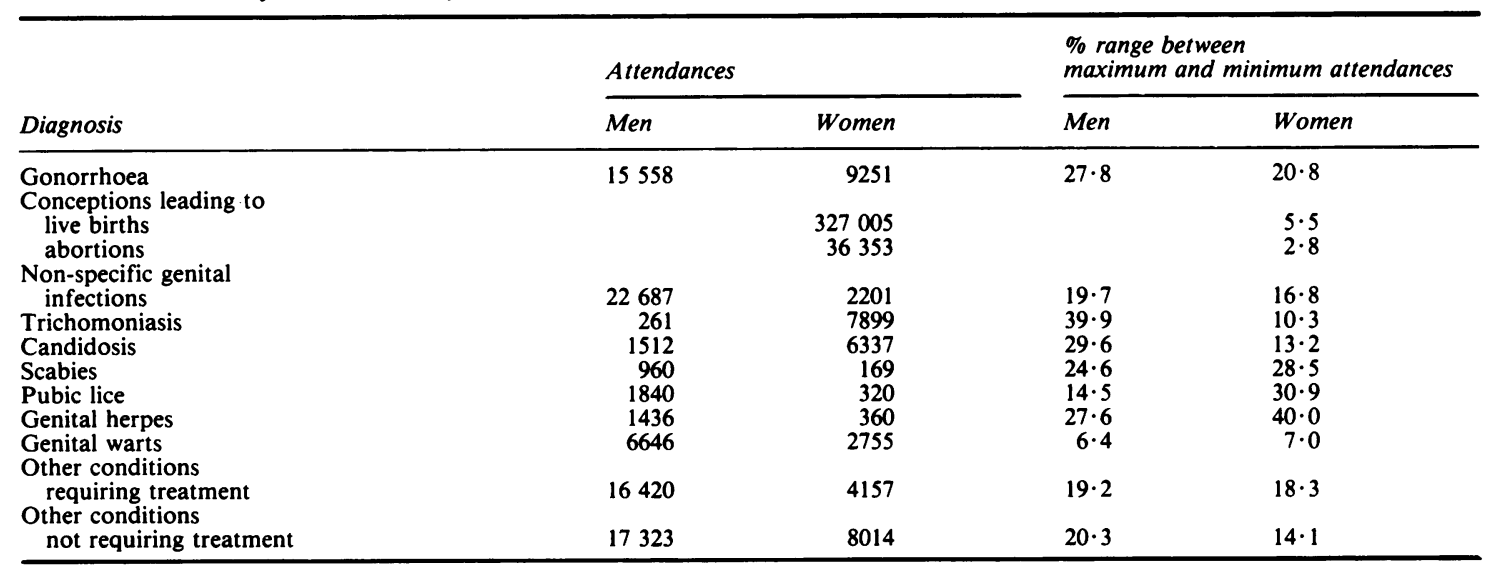

Table 2 Range of seasonal incidence for gonorrhoea and conceptions between men and women

\begin{tabular}{llclc}
\hline \multirow{2}{*}{ 'Control' conditions } & \multicolumn{4}{l}{$\%$ range of incidence (by yearly quarters) } \\
\cline { 2 - 5 } & First & Second & Third & Fourth \\
\hline Gonorrhoea & $\mathbf{8 8 \cdot 9 - 8 9 \cdot 3}$ & $93 \cdot 1-95 \cdot 0$ & $110 \cdot 1-116 \cdot 7$ & $101 \cdot 3-105 \cdot 6$ \\
Conceptions & $97 \cdot 0-100 \cdot 0$ & $101 \cdot 2-102 \cdot 5$ & $100 \cdot 4-101 \cdot 7$ & $98 \cdot 4-98 \cdot 8$ \\
\hline
\end{tabular}




\section{GENITAL HERPES}

Again, few cases of genital herpes infections occurred in women (Figure and Table 1), and the seasonal incidence was unlike that of either gonorrhoea or conceptions, with the minimum attendance in the first and the maximum in the fourth quarters, a range of $40.0 \%$. The seasonal incidence of genital herpes infection in men, however, was similar to that of gonorrhoea, with a range of $27 \cdot 6 \%$.

\section{GENITAL WARTS}

Because of the wide variation in the incubation period of genital warts there was no valid pattern in the seasonal incidence for either sex (Figure and Table 1). The number of male attendances during the first three quarters was steady, with the minimum during the fourth, while the number of female attendances fluctuated from one quarter to the next (Table 1); the ranges were $6 \cdot 4 \%$ and $7 \cdot 0 \%$ respectively.

\section{OTHER CONDITIONS REQUIRING TREATMENT}

$A$ wide variety of usually non-infectious and dissimilar clinical conditions of the genital areas are included under the heading of 'other conditions requiring treatment' (Table 1). Nevertheless, the seasonal incidence in men closely resembled that of gonorrhoea, with the maximum attendance during the third quarter and the minimum in the first, a range of $19 \cdot 2 \%$ (Table 1 ). The seasonal incidence in women was different, however, with the minimum attendance in the first quarter and the maximum in the fourth, a range of $18 \cdot 3 \%$ (Table 1 ).

\section{OTHER CONDITIONS NOT REQUIRING}

TREATMENT

Most of the patients who attended and were found to be free of any sexually transmitted disease and in no need of treatment for any other condition (Table 1) came of their own accord having run a risk of infection. Far fewer attended either as sexual contacts of patients with a sexually transmitted disease and found not to be infected or because they believed, or feared, that they had a disease without being able to cite any particular risk. The seasonal incidence was very similar to that of gonorrhoea, with the maximum attendance during the third quarter and the minimum in the first, a range of $20 \cdot 3 \%$ (Figure). The seasonal incidence for women was also similar, but the range was only $14 \cdot 1 \%$.

\section{Discussion}

A possible explanation for the incidence of diseases associated with promiscuity being at its highest in the third quarter-the months of August, September, and October-has been proffered by Felton (personal communication). First pairings occur in the spring and second and casual ones in the summer; the original partners later rejoin, and about this time symptoms and signs become manifest in one or the other. The highest incidence of conceptions leading to live births or abortions in the second quarter might indicate that they occur mainly in first pairings, especially as there is no peak later in the year.

A similar pattern of disease-incidence for men and women was found in only three conditions, in addition to that of gonorrhoea: non-specific genital infections, other conditions not requiring treatment, and genital candidosis. The seasonal variation in the incidence of the first two conditions was similar to that of gonorrhoea, and this was to be expected. Most cases of non-specific genital infection were associated with promiscuous, sexual relationships by one or other partner, while most patients who attended for follow-up examinations did so after running a risk of infection with a casual partner. The seasonal variation in the incidence of genital candidosis was similar to, but an exaggeration of, that of conceptions. Willmott (1975) did not find any seasonal variation in the incidence of genital yeast infections in women, but he described only 207 cases. The seasonal variation in incidence of both genital candidosis and conceptions could reflect the varying frequency of sexual contact among the population at large, the former being an exaggeration and the latter an underestimation of the incidence; this would agree with the opinions of many clinicians that most cases of genital candidosis are not associated with sexual promiscuity.

For all other conditions differences in the seasonal incidence between the sexes were noted. In the case of genital scabies the minimum number of male attendances occurred during the first and second quarters, with a sharp increase in the third to a maximum number in the fourth. Very few women attended with genital scabies; maximum attendances occurred in the third quarter (which possibly reflects the rising number of infections in men) with a second peak in the first quarter-perhaps an aftermath of the peak in men in the fourth quarter. The high incidence of infections occurring during the cold months might indicate that this infestation is more readily transmitted by close bodily contact indoors than in the open air.

The seasonal incidence of all other conditions in men was similar to that of gonorrhoea, indicating an association with a high rate of sexual promiscuity. The highest incidences of genital herpes and other conditions requiring treatment occurred during the third quarter, with the lowest incidence in the first, 
whereas the highest incidences of trichomoniasis and pubic lice in men occurred in the fourth and second quarters respectively.

The seasonal incidence of these conditions in women was usually lowest during the first quarter-that of trichomoniasis being lowest during the second-with the incidence rising sharply by the third to a peak by the fourth quarter. Neilson (1973) has reported an increase in the frequency with which Trichomonas vaginalis was cultured during the autumn (September, October, and November) in Copenhagen. The dissimilarity between the patterns of attendance of men and women with other conditions requiring treatment can be accounted for by the fact that very few were sexual partners, and they almost certainly represented two completely different populations. The same, however, cannot be said of those attending with infections, trichomoniasis, genital herpes, and pubic lice. As with scabies, over half the attendances by men and women with pubic lice occurred during the first and fourth quarters, which possibly indicates that these infestations are transmitted more easily by close, bodily contact indoors rather than in the open air. Nevertheless, the most probable explanation for the maximum number of female attendances occurring one quarter after that of men is that little contacttracing of patients with these conditions was carried out, most women only attending when symptoms had developed. While men with these conditions were often promiscuous, this was not necessarily true of women.

\section{References}

Cornelius, C. E. (1971). Seasonality of gonorrhoea in the United States. HSMHA Health Reports, 86, 157-160.

Neilson, R. (1973). Trichomonas vaginalis: II Laboratory investigations in trichomoniasis. British Journal of Venereal Diseases, 49, 531-535.

Registrar General for Scotland (1976). Population and vital statistics. Annual Report of the Registrar General for Scotland, p 60. HMSO: Edinburgh.

Schofield, C. B. S. (1975). Sexually Transmitted Diseases, second edition, p 41. Churchill Livingstone: Edinburgh.

Scottish Home and Health Department (1973-76). Scottish abortion statistics. Health Bulletin (Edinburgh), 31, 39; 31, 215; 32,$121 ; 33,167 ; 34,285$

Willmott, F. E. (1975). Genital yeasts in female patients attending a venereal disease clinic. British Journal of Venereal Diseases, 51, 119-122. 